\title{
Active Wing Shaping Control for an Elastic Aircraft
}

\author{
Wilfred Nobleheart \\ Faculty: Animesh Chakravarthy
}

Department of Aerospace Engineering, College of Engineering

\begin{abstract}
This paper discusses active wing shaping control strategies for an elastic aircraft, with highly flexible wings. The objective of in-flight wing shaping is to ensure that the local angle of attack distribution on the wing is optimal at every flight condition so as to result in reduced drag during cruise. The wings are constructed using lightweight materials as a means to reduce aircraft weight - use of such materials ensures that strength and durability is retained, but results in reduced structural rigidity. This increase in structural flexibility leads to a significant increase in the effects of aeroelastic interaction forces and moments, which can lead to important aircraft stability and control issues.
\end{abstract}

\section{Introduction}

Morphing and adaptive aircraft structures possess the ability to adapt and optimize their shape to achieve dissimilar, multi-objective mission roles efficiently and effectively. The introduction of morphing, or shape-changing actuation, to an aircraft will alter the shape and aerodynamics of the vehicle. In some applications, this shape changing option consists of schemes that will modify one or more of the wing area, sweep angle, wing span and airfoil camber, in a manner as to make the aircraft aerodynamically efficient. The focus of this paper is an elastically shaped aircraft that has highly flexible wings, whose shape can be actively controlled in flight. The aircraft concept is biologically inspired and has its origins in the efficient manner in which birds change their wing shapes during flight. Such a wing can accommodate significant curvature, thereby facilitating lower drag. The distributed-flap and distributed-slat wing concept $[1,2]$ is shown in Figure 1 . The output feedback control design technique used in this paper incorporates a multi-objective performance index [1] in that the drag term directly enters the performance index that is to be minimized. The ensuing design equations for minimizing this multi-objective performance index with output feedback are demonstrated. The optimal output feedback gains are used to stabilize the aircraft in the closed loop.

\section{Results and Discussion}

The control problem is to determine flap/slat deflections $\mathrm{u}$ so as to minimize the performance index expressed in (1), with the state equation expressed in (2).

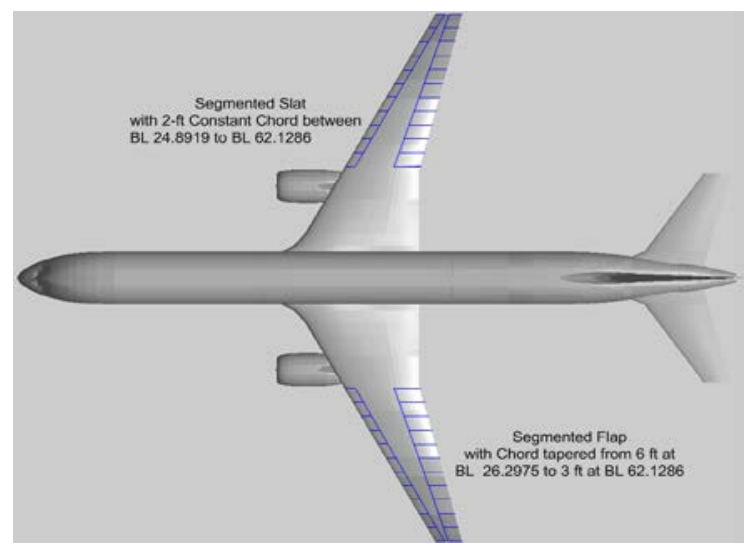

Fig. 1. Distributed Flaps and Slats for Wing Shaping Control

$J=\frac{1}{2} \int_{0}^{\infty}\left(x^{T} Q x+u^{T} R u+q_{d} C_{D}\right) d t(1)$

$\frac{d x}{d t}=A x+B u(2)$

The output feedback design consists of solving equations (3) - (7).

$A_{c}^{T} P_{2}+P_{2} A_{c}+\bar{Q}=0(3)$

$A_{c} S_{2}+S_{2} A_{c}^{T}+\bar{X}=0(4)$

$P_{1}=q_{d} K\left(2 C_{L_{0}} C_{L_{x}}-2 C_{L_{0}} C_{L_{u}} K C-C_{D_{u}} K C\right) A_{c}^{-1}(5)$

$S_{1}=-A_{c}^{-1} x(0)(6)$

$-\frac{1}{2} B^{T} P_{1}^{T} S_{1}^{T} C^{T}-q_{d} C_{0} C_{L_{x}}^{T} S_{1}^{T} C^{T}-\frac{1}{2} q_{d} K C_{D_{U}}^{T} S_{1}^{T} C^{T}-C^{T} P_{2} S_{2} C^{T}$

$+R K C_{2} C^{T}-q_{d} K C_{L_{u}}^{T} C_{L_{x}} S_{2} C^{T}+q_{d} K C_{L_{u}}^{T} C_{L_{u}} C S_{2} C^{T}$

$+q_{d} K C_{D_{u^{2}}}^{T} K S_{2} C^{T}=0(7)$

Where $A_{c}$ represents the closed loop system, and $\mathrm{P}_{2}$ and $S_{2}$ are solutions to the Lyapunov Equations (3)(4). Performance index $J$ computed starting from certain initial gains $K_{0}$, as a function of the iteration number, is plotted in Figure 2. As one can see, for the 
chosen initial gains, the performance index converges to zero.

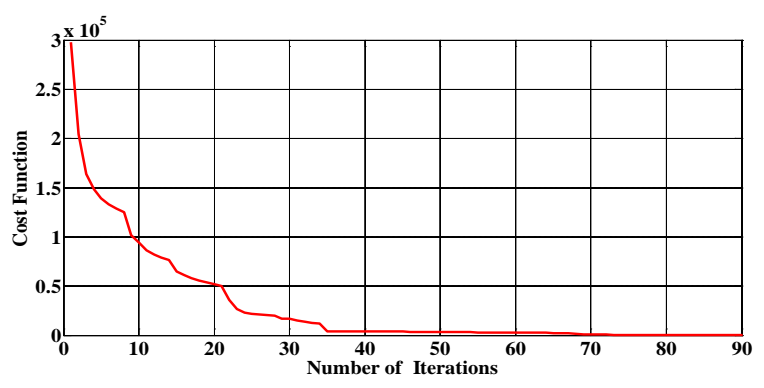

Fig. 2. Performance index J vs. Number of Iterations.

Simulation results with the designed controller gain matrix are demonstrated in Figures 3, 4 and 5. The static output feedback controller ensures that the closed loop system is stable. Figure 3 shows the rigid body states a, asamell as the corresponding elevator deflection egenerated by the controller. As one can see they all converge to zero and eliminate the effect of the initial disturbance.
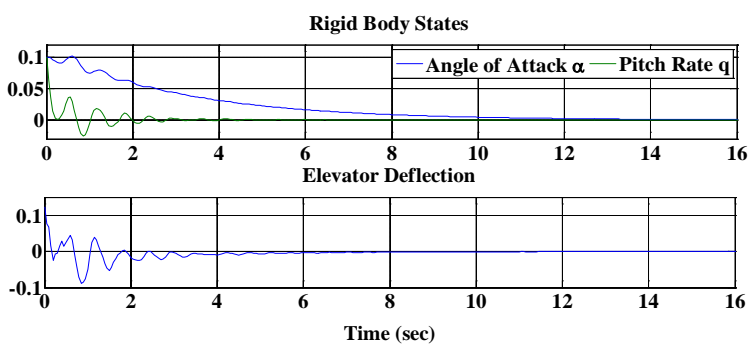

Fig. 3. Rigid Body States and Inputs of the ESAC.

The same can be said for generalized coordinates associated with the elastic states $q_{w}$ and $q_{\theta}$ respectively, and the corresponding elastic inputs generated by the controller, which include the slat and flap deflections $\delta_{s}$ and $\delta_{f}$ respectively, they all converge to zero as shown in Figure 4.

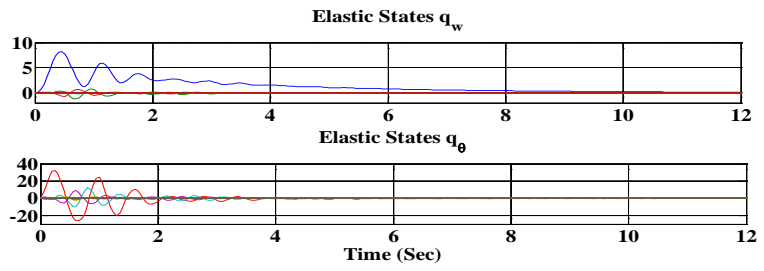

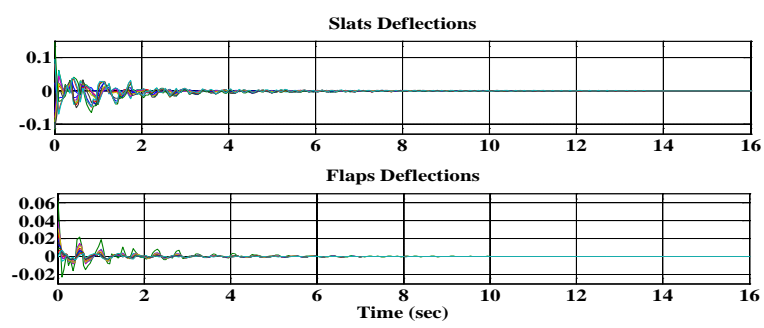

Fig. 4. Rigid Body and Elastic Inputs of the ESAC.

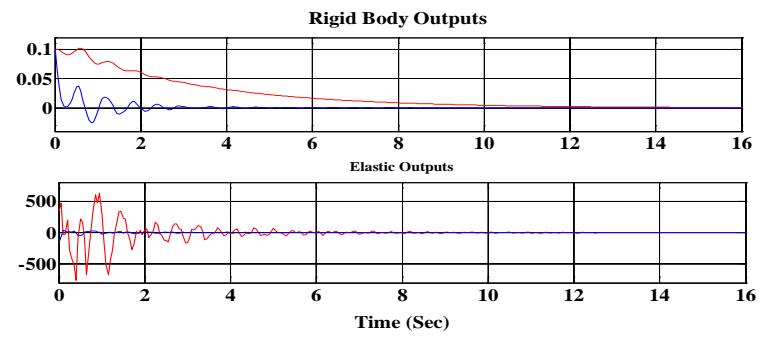

Fig. 5. Aircraft Outputs used for Feedback.

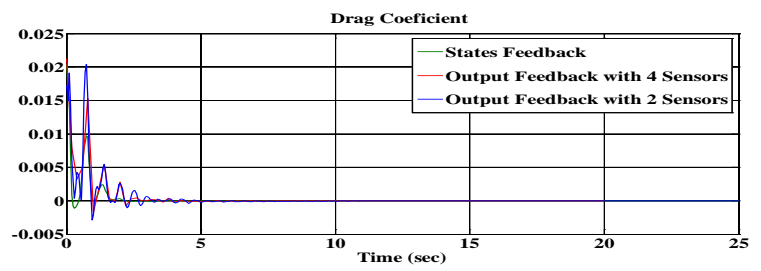

Fig. 6. Drag Coefficient with State Feedback and with Output Feedback.

Figure 6 shows the drag coefficient $C_{D}$ as a function of time with 2 sensors and it is compared to that obtained using the optimal state feedback design and 4 sensors. As one can see, output feedback controller is able to reduce the drag.

\section{Conclusions}

In this paper, the use of static output feedback to determine control laws for active wing shaping of a passenger aircraft conceptualized at NASA is demonstrated. The wing has the ability to change its shape through separate deformations of its leading and trailing edges. A multi-objective performance index that incorporates an additional term explicitly for drag reduction is used, and the corresponding output feedback control law is derived. Simulations of the closed loop aircraft with this control law are performed, and these simulations demonstrate that the output feedback controller is able to stabilize the elastic aircraft. 


\section{Acknowledgement}

This material is based upon work supported by the National Aeronautics and Space Administration NASA EPSCoR at Kansas.

\section{References}

[1] Nguyen, N. and Urnes Sr., James, "Aeroelastic Modeling of Elastically Shaped Aircraft Concept via Wing Shaping Control for Drag Reduction," AIAA Atmospheric Flight Mechanics Conference, August 2012,Minneapolis, MO, AIAA 20124642.

[2] Nobleheart, W., Chakravarthy, A., Nguhen, N. "Active Wing Shaping Control of an Elastic Aircraft.” ACC-2014, Portland, OR. 